\title{
HP MULTIPLIERS AND INEQUALITIES OF HARDY AND LITTLEWOOD
}

\author{
G. I. GAUDRY
}

(Received 13 December 1967)

\section{Introduction}

Consider the classical Hardy spaces $H^{p}(T)(1 \leqq p \leqq \infty)$ on the unit circle $T$. We shall ignore completely the fact that the elements of $H^{p}(T)$ can be extended via the Poisson formula to certain types of functions analytic inside the unit disc. For our purposes, $H^{p}(T)$ is the closed ideal in $L^{p}(T)$ consisting of those functions $f \in L^{p}(T)$ for which $\hat{f}(n)=0$ $(n=-1,-2, \cdots)$.

Let $\varphi$ be a function (complex-valued) defined on $Z$ ( $Z$ stands for the group of the integers; $Z_{+}$for the subsemigroup of $Z$ consisting of the nonnegative integers.) We say that $\varphi$ is a multiplier from $H^{p}(T)$ to $H^{q}(T)$ if, whenever $f \in H^{p}(T)$, $\varphi \hat{f}$ is the Fourier transform of an element of $H^{\alpha}(T)$. More generally, if $S_{1}(T)$ and $S_{2}(T)$ are sets of measures (or distributions) on $T$ ( $T$ may be replaced by other groups), we may define analogously what is meant by saying that $\varphi$ is a multiplier from $S_{1}(T)$ to $S_{2}(T)$.

A considerable amount is known about the properties and misbehaviour of multipliers from $L^{p}$ to $L^{q}$ (even when these spaces are defined over quite general groups). Our purpose in $\S 2$ is to show by means of simple counterexamples that the multipliers from $H^{p}(T)$ to $H^{q}(T)$ are, when $p=1$, considerably more pathological than those from $L^{p}(T)$ to $L^{q}(T)$, and are just as pathological as the latter when $p>1$. Specifically, we construct multipliers $\varphi$ from $H^{p}(T)$ to $H^{q}(T)(1 \leqq p \leqq \infty, 1 \leqq q<\infty)$ which have the property that $\varphi$ restricted to $Z_{+}$is not the restriction to $Z_{+}$of any Fourier-Stieltjes transform. Recall that the multipliers from $L^{p}(T)$ to $L^{q}(T)$ when $p=1$ and $1 \leqq q<\infty$ are precisely (i) the Fourier-Stieltjes transforms when $q=1$; and (ii) the Fourier transforms of elements of $L^{q^{\prime}}(T)$ when $1<q<\infty$. Cf. Edwards [1], Chapter 16.

Next, in $\S 3$, we consider the following general situation. Let $G$ be a compact Abelian group with ordered dual $X . X_{+}$will stand for the set of nonnegative elements of $X$. One may define the Hardy spaces on $G$ relative to normalised Haar measure on $G$ and the order on $X$ in the obvious way. Cf. Rudin [4], Chapter 8. Let $A(G)$ be the space of continuous functions 
$f$ on $G$ for which $\hat{f}(\chi)=0$ for $\chi \in X_{-}\left(=X \backslash X_{+}\right)$and $\sum|\hat{f}(\chi)|<\infty$. In Theorem 3.2 we characterise the multipliers from $H^{1}(G)$ to $A(G)$. This yields a generalisation of the classical Hardy inequality (Hoffman [2], pp. $70-71)$ :

For all $f \in H^{1}(T)$,

$$
\sum_{1}^{\infty} \frac{1}{n}|\hat{f}(n)| \leqq\left.\pi|| f\right|_{1}
$$

In all that we do in $\S \S 3-5$ the fundamentally important tools are: (i) the general factorisation theorem for $H^{1}$ functions (Theorem 3.1) due to Helson and Lowdenslager; and (ii) the general M. Riesz theorem proved by Helson (Theorem 4.2). See Rudin [4], Chapter 8.

Using the results of Helson and Lowdenslager, we generalise the Calderón-Zygmund interpolation theorem for $H^{p}$ spaces in $\S 4$. The proof is basically the same as that given by Zygmund in the case $G=T$ (Zygmund [7], Chapter XII (3.9)) even though the argument presented by Zygmund depends heavily on the fact that the functions in $H^{p}(T)$ can be thought of as analytic functions in the unit disc.

Finally in $\S 5$ we use the interpolation theorem and the generalised Hardy inequality to prove the generalised Hardy-Littlewood inequality. In the classical case, the Hardy-Littlewood inequality states that for every $p: 1<p \leqq 2$, there exists a constant $A_{p}$ such that

$$
\left(\sum(1+|n|)^{p-2}|\hat{f}(n)|^{p}\right)^{1 / p} \leqq A_{p}\|f\|_{p} \quad\left(f \in L^{p}(T)\right)
$$

(Zygmund [7], (3.19); Edwards [1], 13.11.1).

\section{Multipliers from $H^{p}(T)$ to $H^{q}(T)$}

Let $\left(n_{k}\right)_{1}^{\infty}$ be an increasing sequence of positive integers. We say that $\left(n_{k}\right)$ is a Hadamard sequence if there exists a real number $r>1$ such that $n_{k+1} \geqq r n_{k}$ for all $k$. We say that $\left(n_{k}\right)$ is a Paley sequence if it is a finite union of Hadamard sequences. The reason for the adoption of the latter terminology is contained in the following useful theorem of Paley and Rudin (Rudin [5], Theorem 1). Then

2.1 THEOREM. Let $\left(n_{k}\right)_{1}^{\infty}$ be an increasing sequence of positive integers.

$$
\sum_{k=1}^{\infty}\left|\hat{f}\left(n_{k}\right)\right|^{2}<+\infty
$$

for all $f \in H^{1}(T)$ if and only if $\left(n_{k}\right)$ is a Paley sequence.

Observe that any Paley sequence, being a finite union of Hadamard sequences, is a Sidon set and is hence of type $A(p)$ for all $p: 0<p<\infty$. 
For an explanation of the terminology and a proof of this assertion see Rudin [6].

We shall need one further result, viz. Rudin's extension of the F. and M. Riesz theorem (Rudin [6], 5.7).

2.2 Theorem. Let $S$ be a subset of $Z_{+}$which is of type $A(1)$, and suppose that $\mu$ is a measure on $T$ such that $\mu(n)=0$ for $n \in Z_{+} \backslash S$. Then $\mu$ is absolutely continuous with respect to Lebesgue measure.

We now present our counterexample concerning multipliers.

2.3 Theorem. Suppose that $1 \leqq p \leqq \infty, 1 \leqq q<\infty$. Then there exist multipliers $\varphi$ from $H^{p}(T)$ to $H^{q}(T)$ which have the property that $\varphi \mid Z_{+}$is not the restriction to $Z_{+}$of any Fourier-Stieltjes transform.

Proof. Let $S=\left(n_{k}\right)_{1}^{\infty}$ be any Paley sequence in $Z_{+}$and let $\left(c_{k}\right)$ be a bounded sequence such that $c_{k}$ does not tend to zero as $k \rightarrow+\infty$. Define

$$
\varphi(n)=\left\{\begin{array}{l}
c_{k} \\
0
\end{array}\right.
$$

$$
\begin{aligned}
& n=n_{k} \\
& n \neq n_{k} .
\end{aligned}
$$

By Theorem 2.1, if $f \in H^{p}(T)\left(C H^{1}(T)\right)$,

$$
\sum_{n \in Z}|\hat{f}(n) \varphi(n)|^{2}<+\infty \text {. }
$$

$(\hat{f}(n) \varphi(n))_{n \in Z}$ vanishes outside a Sidon set, and is an element of $\ell^{2}(Z)$. Hence (Rudin [6], 3.1), it is the transform of a function which belongs to $L^{q}(T)$ for every $q: 1 \leqq q<\infty$. So $\varphi$ is a multiplier from $H^{p}(T)$ to $H^{q}(T)$. On the other hand, Theorem 2.2 shows that if $\varphi \mid Z_{+}$is the restriction to $Z_{+}$ of a Fourier-Stieltjes transform $\hat{\mu}$, then $\mu$ is absolutely continuous with respect to Lebesgue measure, so that $\hat{\mu}(n) \rightarrow 0$ as $|n| \rightarrow+\infty$. But by hypothesis, $c_{k}$ does not tend to zero as $k \rightarrow+\infty$. Hence $\varphi \mid Z_{+}$is not the restriction to $Z_{+}$of any Fourier-Stieltjes transform.

REMarks. (i) In a recent note, Meyer [3] has exhibited an example of a multiplier of $H^{1}(T)$. His example is constructed as follows. Let $\left(x_{k}\right)_{k=0}^{\infty}$ be a sequence of positive real numbers, $x_{0}>0$, such that for some $r>1$, $x_{k+1} \geqq r x_{k}$ for all $k$. Let $f$ be a function in $L^{\mathbf{1}}(R)$ such that $f(x)=0$ for $x \notin\left[-x_{0}, x_{0}\right]$. Then if $\left(c_{k}\right)$ is any bounded sequence, the function $\varphi$ defined by

$$
\varphi(n)= \begin{cases}\sum_{\mathbf{1}}^{\infty} c_{k} \hat{f}\left(n-x_{k}\right), & n \geqq 0 \\ 0, & n<0\end{cases}
$$

is a multiplier from $H^{1}(T)$ to $H^{1}(T)$. It is, however, not very difficult to show that any such $\varphi$ must be bounded and must vanish outside a Paley sequence; so Meyer's example is a particular case of the construction we 
have given in Theorem 2.3. (Whilst the argument presented by Meyer is considerably more complicated than the rather simple one we have presented, it should be noted that Meyer needs the careful estimate of the multiplier norm of $\varphi$ which his argument yields for later application to a problem in spectral synthesis; it appears that our simple argument does not yield such information.)

(ii) In the same note, Meyer proves that if $\psi$ is a continuous function on $R$, and if $H^{1}(R)$ is defined as the closed ideal in $L^{1}(R)$ consisting of those functions $f$ whose Fourier transforms vanish outside $[0,+\infty)$, then $\psi$ is a multiplier of $H^{1}(R)$ if and only if $(\psi(n / K))_{n \geqq 1}$ is a multiplier of $H^{1}(T)$ for every positive integer $K$. It should be observed that by combining this theorem with the fact that the Fourier-Stieltjes transforms of measures on $T$ are precisely the restrictions to $Z$ of the Fourier-Stieltjes transforms of bounded measures on $R$ (Rudin [4], 2.7.2) one can exhibit multipliers $\psi$ of $H^{1}(R)$ which have the property that $\psi \mid[0,+\infty)$ is not the restriction to $[0,+\infty)$ of any Fourier-Stieltjes transform.

The situation when $q=\infty$ is very simple, as is shown by the following theorem.

2.4 TheOREM. The multipliers from $H^{p}(T)$ to $H^{\infty}(T)(1 \leqq p \leqq \infty)$ are precisely (i) the functions $\varphi$ such that $\varphi \mid Z_{+}$is the restriction to $Z_{+}$of a FourierStieltjes transform when $p=\infty$; (ii) the functions $\varphi$ such that $\varphi \mid Z_{+}$is the restriction to $Z_{+}$of the Fourier transform of an element of $L^{p^{\prime}}(G)$ when $1 \leqq p<\infty$.

Proof. (i) Clearly, any $\varphi$ of the stated form is a multiplier. Suppose conversely that $\varphi$ is a multiplier from $H^{\infty}(T)$ to $H^{\infty}(T)$, and consider the corresponding operator $L$ from $H^{\infty}(T)$ to $H^{\infty}(T)$ defined by $(L f)^{\wedge}=\varphi \hat{f}$. $L$ is linear and, by the closed graph theorem, continuous. So

$$
\|L f\|_{\infty} \leqq K\|f\|_{\infty}
$$

for all $f \in H^{\infty}(T)$ and some finite constant $K$. $L$ maps the set of 'analytic' trigonometric polynomials into trigonometric polynomials. Consider then the mapping $\sigma: f \rightarrow L f(0)$ on the set of analytic trigonometric polynomials, the latter space being given the sup-norm topology. By (2.4.1) $\sigma$ is continuous. By the Hahn-Banach theorem, there exists a measure $\mu$ on $T$ such that

$$
L f(0)=\mu\left(f_{\mathrm{v}}\right)
$$

for all analytic trigonometric polynomials, where $f_{v}(x)=f(-x)$. If in (2.4.2) we take successively $f=1, e^{i x}, e^{2 i x}, \cdots$, we get $\varphi\left|Z_{+}=\hat{\mu}\right| Z_{+}$.

(ii) The proof when $p<\infty$ is entirely similar to that for the case $p=\infty$. 
REMARK. This theorem generalises in an obvious way to the case where $T$ is replaced by a compact Abelian group with ordered dual.

\section{Multipliers from $H^{1}(G)$ to $A(G)$ : Hardy's inequality}

Let $G$ be a compact Abelian group with ordered dual $X$ (so that $G$ is connected: Rudin [4], Chapter 8). Write $X_{+}$for the semigroup consisting of the nonnegative elements of $X$, and let $H^{p}(G)$ be the Hardy spaces defined relative to normalised Haar measure $d x$ on $G$ and the given order on $X$. Helson and Lowdenslager extended the classical factorisation theorem for $H^{1}$ functions to this general situation by using a Hilbert space argument. We state their result in the following form. (See Rudin [4], 8.4.4 and its proof.)

3.1 Theorem. Suppose that $f \in H^{1}(G)$ and $\hat{f}(0) \neq 0$. Then there exist functions $f_{1}, f_{2}$ in $H^{2}(G)$ with $\left|f_{i}\right|^{2}=|f|(i=1,2)$ and $f=f_{1} f_{2}$. In particular, $\left\|f_{i}\right\|_{2}^{2}=\|f\|_{1}$ for each $i$.

Let $A(G)$ be the subspace of $H^{1}(G)$ consisting of those continuous functions $f$ for which $\sum_{\chi \in X}|\hat{f}(\chi)|<+\infty$. We norm $A(G)$ by setting $\|f\|=\sum|\hat{f}(\chi)|$.

The following result generalises the classical Hardy inequality and characterises the multipliers $\varphi$ from $H^{1}(G)$ to $A(G)$.

3.2 THEOREM. The multipliers from $H^{1}(G)$ to $A(G)$ are precisely those functions $\varphi$ on $X$ with the property that $\varphi \mid X_{+}$and $|\varphi| \mid X_{+}$are both the restrictions to $X_{+}$of the Fourier transforms of elements of $L^{\infty}(G)$. When $\varphi$ is such a function on $X$, there exists a constant $K$ such that

$$
\sum|\varphi(\chi) \hat{f}(\chi)| \leqq K\|f\|_{1} \quad\left(f \in H^{1}(G)\right)
$$

Proof. The proof is implicit in the traditional proof of Hardy's inequality (as presented for example by Hoffman [2], pp. 70-71).

Suppose that $\varphi$ is a multiplier. Then the same is true of $|\varphi|$, so we may as well suppose throughout that $\varphi$ is nonnegative. When $\varphi \geqq 0$ is a multiplier, the mapping $L$ defined by

$$
(L f)^{\wedge}=\varphi \hat{f}
$$$$
\left(f \in H^{1}(G)\right)
$$

defines a linear mapping from $H^{1}(G)$ to $A(G)$. The closed graph theorem shows that this map is continuous. So a function $\varphi \geqq 0$ on $X$ is a multiplier if and only if there exists a constant $K>0$ such that

$$
\sum \varphi(\chi)|\hat{f}(\chi)| \leqq K|| f \|_{1}
$$$$
\left(f \in H^{1}(G)\right) .
$$

Clearly, if $\varphi$ is a multiplier, the mapping $f \rightarrow \sum \varphi(\chi) \hat{f}(\chi)$ defines a continuous linear form on $H^{1}(G)$. It follows from the Hahn-Banach theorem that there exists a function $\Phi \in L^{\infty}(G)$ such that 


$$
\sum \varphi(\chi) \hat{f}(\chi)=\int_{G} \Phi(-x) f(x) d x
$$

whence $\varphi(\chi)=\hat{\Phi}(\chi)\left(\chi \in X_{+}\right)$.

For the converse, let $\Phi$ be an element of $L^{\infty}(G)$ with $\phi(\chi) \geqq 0\left(\chi \in X_{+}\right)$. Suppose $\varphi(\chi)=\hat{\Phi}(\chi)$ on $X_{+}$and (say) $\varphi(\chi)=0$ on $X_{-}$. For each finite subset $S \subset X$, write $\Psi_{S}$ for a function in $L^{\mathbf{1}}(G)$ with the following properties: (i) $\hat{\Psi}_{S}(\chi)=1 \quad(\chi \in S)$; (ii) $\hat{\Psi}_{S} \geqq 0$; (iii) $\left\|\hat{\Psi}_{S}\right\|_{1} \leqq 2$; (iv) $\hat{\Psi}_{S}$ has finite support. Such a function $\Psi_{S}$ always exists (Rudin [4], 2.6.8). An application of Hölder's inequality yields

$$
\begin{aligned}
\left|\sum \varphi(\chi) \hat{\Psi}_{S}(\chi) \hat{f}(\chi)\right| & =\left|\int \Phi(x) f * \Psi_{S}(-x) d x\right| \\
& \leqq\|\Phi\|_{\infty}\left\|f * \Psi_{S}\right\|_{1} \\
& \leqq 2\|\Phi\|_{\infty}\|f\|_{1}
\end{aligned}
$$

for all $f \in H^{1}(G)$.

Suppose first of all that $f \in H^{1}(G)$ and that $\hat{f}(0) \neq 0$. Then by Theorem 3.1, we can write $f=g h$, where $g, h \in H^{2}(G)$, and $\|g\|_{2}^{2}=\|h\|_{2}^{2}=\|f\|_{1}$. Write $G$ and $H$ for the functions defined (d'après Riesz-Fischer) by $\hat{G}(\chi)=|\hat{g}(\chi)|, \hat{H}(\chi)=|\hat{h}(\chi)|$. Then $\|G\|_{2}^{2}=\|H\|_{2}^{2}=\|f\|_{1}$. Replacing $f$ in $(3.2 .3)$ by $G H$, and observing that $(G H)^{\wedge}(\chi) \geqq|\hat{f}(\chi)|$, we get

$$
\begin{aligned}
\sum_{x \in S} \varphi(\chi)|\hat{f}(\chi)| & \leqq \sum_{x \in X} \varphi(\chi) \hat{\Psi}_{S}(\chi)(G H)^{\wedge}(\chi) \\
& \leqq 2\|\Phi\|_{\infty}\|G H\|_{1} \\
& \leqq 2\|\Phi\|_{\infty}\|f\|_{1}
\end{aligned}
$$

the last step by the Gauchy-Schwarz inequality. But (3.2.4) is true for any finite subset $S$ of $X$. Hence $\sum_{\chi \in X} \varphi(\chi)|\hat{f}(\chi)|<+\infty$.

When $f \in H^{1}(G)$ and $\hat{f}(0)=0$, replace $f$ in (3.2.4) by $f+1 / n$ and let $n \rightarrow+\infty$. So we deduce that $\varphi$ is a multiplier from $H^{1}(G)$ to $A(G)$. This completes the proof of the theorem.

3.3 CoRollary. If $\varphi \geqq 0$ on $X_{+}$is the restriction to $X_{+}$of the transform of a function in $L^{\infty}(G)$, the same is true of $\psi$ and $|\psi|$ for any function $\psi$ defined on $X_{+}$for which $|\psi| \leqq \varphi$.

3.4 COROLLARY. If $\varphi \geqq 0$ on $X$ has the property that $\varphi \mid X_{+}$is the restriction to $X_{+}$of the Fourier transform of a function in $L^{\infty}(G)$, then for any function $\Psi$ in $L^{\infty}(G)$ for which $|\hat{\Psi}(\chi)| \leqq \varphi(\chi)\left(\chi \in X_{+}\right)$, we have the Parseval formula

$$
\sum \hat{\Psi}(\chi) \hat{f}(\chi)=\int_{G} f(x) \Psi(-x) d x \quad\left(f \in H^{1}(G)\right)
$$

the series being absolutely convergent (rather than simply summable).

Remarks. (i) The classical Hardy inequality results when one takes $\Phi\left(e^{i x}\right)=i(\pi-x)$ on $G=T$ in Theorem 3.2. 
(ii) Consider the multipliers from $H^{p}(G)$ to $A(G)$ when $1<p \leqq \infty$ (regarded as functions on $X_{+}$). When $p \geqq 2$, it is not difficult to see that these are precisely the functions $\varphi \in \ell^{2}\left(X_{+}\right)$. One half of the proof is obvious. For the other half, suppose $\varphi$ is a multiplier from $H^{p}(G)$ to $A(G)$ (with $p$ unrestricted); then $\varphi$ is a multiplier from $C_{A}(G)$ into $A(G) . \varphi$ is therefore on $X_{+}$an element of $\ell^{2}\left(X_{+}\right)$(Rudin [4], 8.7.8).

On the other hand, when $1<p<2$ and $G=T$, there are multipliers $\varphi$ from $H^{p}(G)$ into $A(G)$ which are not in $\ell^{a}\left(X_{+}\right)$for any $q<2$. This is easily seen by choosing $\varphi \notin \bigcup_{q<2} \ell^{q}\left(X_{+}\right), \varphi \in \ell^{2}\left(X_{+}\right)$with $\varphi$ vanishing off a Paley sequence. The problem of characterising the multipliers from $H^{p}(G)$ into $A(G)$ when $1<p<2$ would appear to remain open.

\section{The Riesz-Thorin theorem for $H^{p}$ spaces}

The well known Riesz-Thorin convexity theorem has the following n-dimensional formulation (Zygmund [7], Chapter XII (3.3)).

4.1 Theorem. Let $p_{11}, \cdots, p_{n 1}, p_{12}, \cdots, p_{n 2}$ and $q_{1}$ and $q_{2}$ be real numbers in the range $[1, \infty]$. Let $E$ and $E_{1}, \cdots, E_{n}$ be measure spaces with measures $\mu$ and $v_{1}, \cdots, v_{n}$ respectively. Suppose that $T$ is an operator defined on all $n$-tuples $\left(h_{1}, \cdots, h_{n}\right)$ of functions $h_{i}$, each $h_{i}$ being $v_{i}$-simple, that $T$ has range in $L^{q_{1}} \cap L^{q_{2}}(E ; \mu)$ and that $T$ is multilinear. If $T$ is of types $\left(p_{11}, \cdots, p_{n 1} ; q_{1}\right)$ and $\left(p_{12}, \cdots, p_{n 2} ; q_{2}\right)$ :

$$
\left\|T\left(h_{1}, \cdots, h_{n}\right)\right\|_{a_{i}, \mu} \leqq M_{i} \prod_{j=1}^{n}\left\|h_{j}\right\|_{p_{j i}, \nu_{j}} \quad(i=1,2),
$$

then for $0<t<1$, there exists a constant $M_{t}$ such that

$$
\left\|T\left(h_{1}, \cdots, h_{n}\right)\right\|_{a, \mu} \leqq M_{t} \prod_{j=1}^{n}\left\|h_{j}\right\|_{p_{j}, \nu_{j}}
$$

for $\left(p_{1}, \cdots, p_{n} ; q\right)$ defined by

$$
\frac{1}{q}=\frac{t}{q_{1}}+\frac{1-t}{q_{2}}, \quad \frac{1}{p_{i}}=\frac{t}{p_{i 1}}+\frac{1-t}{p_{i 2}} \quad(i=1, \cdots, n) .
$$

Calderón and Zygmund applied Theorem 4.1 to interpolation in $H^{p}$ spaces over the circle group. The relevant theorem, given in (3.9), Chapter XII, of Zygmund [7], relies heavily on the fact that they are working in the context of the circle group, and so can regard the functions in $H^{p}$ as analytic functions in the disc. We now show that by using Theorem 3.1 in conjunction with Helson's version of the M. Riesz theorem, one can prove the interpolation theorem for $H^{p}$ spaces over the general groups we are concerned with. First we record the enunciation of the general M. Riesz theorem. 
4.2 Theorem. (M. Riesz, Helson) (Rudin [4], 8.7.2) For each trigonometric polynomial $f$ on $G$ define the analytic contraction $f_{c}$ of $f$ by $\hat{f}_{\mathrm{c}}(\chi)=0$ $\left(\chi \in X_{-}\right), \hat{f}_{c}(\chi)=\hat{f}(\chi) \quad\left(\chi \in X_{+}\right)$. Then for each $p$ satisfying $1<p<\infty$, there exists a constant $C_{p}$ such that

$$
\left\|f_{c}\right\|_{p} \leqq C_{p}\|f\|_{p}
$$

for all trigonometric polynomials $f$. Hence the mapping $f \rightarrow f_{c}$ can be extended continuously to the whole of $L^{p}(G)$.

4.3 Theorem. (Riesz-Thorin theorem for $H^{p}$ spaces) Let $G$ be a compact Abelian group with ordered dual $X$ and $(E ; \mu)$ a measure space.

Suppose that $1 \leqq p_{i}<\infty, 1 \leqq q_{i} \leqq \infty$ for $i=1,2$, and that $T$ is a linear operator defined on the analytic trigonometric polynomials on $G$ and with range in $L^{q_{1}} \cap L^{q_{2}}(E ; \mu)$. Suppose further that there exist constants $M_{1}$ and $M_{2}$ such that

$$
\|T f\|_{q_{i}, \mu} \leqq M_{i}|| f \|_{p_{i}}
$$

$(i=1,2)$ for all analytic trigonometric polynomials $f$. Then if $0<t<1$, and

$$
\frac{1}{p}=\frac{t}{p_{1}}+\frac{1-t}{p_{2}}, \frac{1}{q}=\frac{t}{q_{1}}+\frac{1-t}{q_{2}},
$$

there exists a constant $M$ such that

$$
\|T f\|_{\boldsymbol{q}, \mu} \leqq M\|f\|_{p}
$$

for all analytic trigonometric polynomials $f$.

Proof. Suppose without loss of generality that $p_{1} \leqq p_{2}$. Observe that $T$ can be extended uniquely to the whole of $H^{p_{1}}(G)$ so that (4.3.1) continues to hold for all $f \in H^{p_{i}}(G)$ (for both pairs of exponents).

The idea of the proof is as follows. The mapping $f \rightarrow f_{c}$ defined say on the set of simple functions on $G$, is continuous in the $L^{p}$ norm when $1<p<\infty$, but it is not continuous in the $L^{1}$ norm. If both $p_{1}$ and $p_{2}$ were greater than one, we could prove the present theorem simply by applying the one-dimensional form of the Riesz-Thorin theorem to the map $T^{\prime}$ defined on $L^{p_{i}}(G)$ by $T^{\prime}(f)=T\left(f_{c}\right)$. This map would then be continuous for the pairs $\left(p_{i}, q_{i}\right)(i=1,2)$ by virtue of the M. Riesz theorem.

It is in order to avoid difficulties when either $p_{1}=1$ or $p_{2}=1$ that we use the $n$-dimensional (in fact 2 -dimensional) form of the Riesz-Thorin theorem.

Observe that $2 p_{i}>1$ for each $i$. Let $\left(h_{1}, h_{2}\right)$ be an ordered pair of functions each of which is a simple function on $G$. Define $T^{\prime}$ on the set of all such ordered pairs as follows: 


$$
T^{\prime}\left(h_{1}, h_{2}\right)=T\left(h_{1 \mathrm{c}} h_{2 \mathrm{c}}\right)
$$

$T^{\prime}$ is defined: for $h_{1 c}, h_{2 c} \in H^{2 p_{i}}(i=1,2)$ since $2 p_{i}>1$ and therefore $h_{1 c} h_{2 c} \in H^{p_{i}}(G)$ by Hölder's inequality. Since $2 p_{i}>1$, it is obvious from the hypotheses concerning $T$ and Hölder's inequality that $T^{\prime}$ (which is bilinear) is of type $\left(2 p_{1}, 2 p_{1} ; q_{1}\right)$ and of type $\left(2 p_{2}, 2 p_{2} ; q_{2}\right)$. By Theorem $4.1, T^{\prime}$ is of type $(2 p, 2 p ; q)$ : i.e. there exists a constant $M$ such that

$$
\begin{aligned}
\left\|T\left(h_{1 c} h_{2 c}\right)\right\|_{\boldsymbol{\alpha}, \mu} & =\left\|T^{\prime}\left(h_{1}, h_{2}\right)\right\|_{\alpha, \mu} \\
& \leqq M\left\|h_{1}\right\|_{2 p}\left\|h_{2}\right\|_{2 p}
\end{aligned}
$$

for all pairs $\left(h_{1}, h_{2}\right)$ of simple functions on $G$. Since $p<\infty, T^{\prime}$ can be extended to the whole of $L^{2 p}(G) \times L^{2 p}(G)$ whilst preserving (4.3.3).

If now $f$ is an analytic trigonometric polynomial and $\hat{f}(0) \neq 0$, we can write $f=f_{1} f_{2}$ where $f_{i} \in H^{2}(G)$ and $\left|f_{i}\right|^{2}=|f|(i=1,2)$. Since $f$ is a trigonometric polynomial, we have $f \in L^{p}(G)$; and since $\left|f_{i}\right|^{p p}=|f|^{p}$, it follows that $f_{i} \in H^{2 p}(G)$ and that $\left\|f_{i}\right\|_{2 p}^{2}=\|f\|_{p}$. Clearly $f_{i} \in H^{2}(G)$ implies $f_{i c}=f_{i}$ for each $i$. It follows from (4.3.3) that

$$
\begin{aligned}
\left\|T\left(f_{1 c} f_{2 c}\right)\right\|_{q, \mu} & =\|T f\|_{\alpha, \mu} \leqq M\left\|f_{1}\right\|_{2 p}\left\|f_{2}\right\|_{2 p} \\
& =M\|f\|_{p}
\end{aligned}
$$

for all analytic trigonometric polynomials $f$ with $\hat{f}(0) \neq 0$. When $\hat{f}(0)=0$, simply replace $f$ by $f+1 / n$ in the above, and let $n \rightarrow+\infty$. So we have established (4.3.2).

\section{The generalised Hardy-Littlewood inequality}

With Theorem 4.3 at our disposal, we can now easily prove the generalised Hardy-Littlewood inequality.

5.1 Theorem. Let $G$ be a compact Abelian group with ordered dual $X$. Then if $\Phi \in L^{\infty}(G)$ and $\hat{\Phi}(\chi) \geqq 0$ on $X_{+}$, there exists, for each $p$ in the range $1<p \leqq 2$, a constant $A_{p}$ such that

for all $f \in L^{p}(G)$.

$$
\left(\sum_{\chi \in X_{+}} \hat{\Phi}(\chi)^{2-p}|\hat{f}(\chi)|^{p}\right)^{1 / p} \leqq A_{p}\|f\|_{p}
$$

Proof. Let $E$ be the measure space $X_{+}$with the discrete measure $\mu$ : $\mu(\{\chi\})=\hat{\boldsymbol{\Phi}}(\chi)^{2}$. The generalised Hardy inequality (Theorem 3.2. Cf. (3.2.1)) asserts that the mapping $T: f \rightarrow(\hat{f} \mid \hat{\Phi}) \mid X_{+}$is continuous from $H^{1}(G)$ to $L^{1}(E ; \mu)$. (We adopt the usual convention that $0 \cdot \alpha=0$ for any nonnegative extended real number $\alpha$.) The Plancherel theorem asserts that this same map is continuous from $H^{2}(G)$ to $L^{2}(E ; \mu)$. $T$ is clearly 
linear. It follows from Theorem 4.3 that $T$ is continuous from $H^{p}(G)$ to $L^{p}(E ; \mu)$ : i.e. there exists a constant $M_{p}$ such that

$$
\left(\sum_{\chi \in X_{+}} \hat{\Phi}(\chi)^{2-p}|\hat{f}(\chi)|^{p}\right)^{1 / p} \leqq M_{p}\|f\|_{p} \quad\left(f \in H^{p}(G)\right) .
$$

But for $1<p \leqq 2$, the mapping $g \rightarrow g_{e}$ is continuous from $L^{p}(G)$ to $H^{p}(G)$ (Theorem 4.2); so there exists a constant $A_{p}$ such that (5.1.1) holds. This proves the theorem.

\section{Acknowledgment}

This research was carried out while the author held an Australian National University Postdoctoral Travelling Fellowship.

Note added in proof. Professor Goes has recently brought to my attention the fact that, for the case where $G$ is the circle group, Theorem 3.2 has been proved previously by R. P. Boas (Journal d'Analyse Mathématique, 10 (1962), 253-271) and independently by James Caveny.

\section{References}

[1] R. E. Edwards, Fourier series: a modern introduction, Vols I, II. (Holt, Rinehart and Winston, New York, 1967).

[2] Kenneth Hoffman, Banach spaces of analytic functions, (Prentice-Hall, Englewood Cliffs $1962)$.

[3] Yves Meyer, 'Multiplicateurs des coefficients de Fourier des fonctions intégrables analytiques', C. R. Acad. Sci. Paris, Sér. A-B, 263 (1966), A385-A387.

[4] Walter Rudin, Fourier analysis on groups, (Interscience, New York 1962).

[5] Walter Rudin, 'Remarks on a theorem of Paley', J. London Math. Soc, 32 (1957), $307-311$.

[6] Walter Rudin, 'Trigonometric series with gaps', J. Math. and Mech., 9 (1960), 203-228.

[7] Antoni $Z$ ygmund, Trigonometric series, Vol. II, (Cambridge University Press 1959).

Mathematics Institute

University of Warwick

Coventry 